\title{
A New Gas Discharge Process for Preparation of Non-fouling Surfaces on Biomaterials
}

\author{
M.-S. Sheu, A. S. Hoffman \\ Center for Bioengineering, FL-20, University of Washington, Seattle, WA 98195, USA
}

\section{J. G. A. Terlingen \& J. Feijen}

Department of Chemical Engineering, University of Twente, PO Box 217, 7500 AE Enschede, The Netherlands

\begin{abstract}
A non-fouling surface containing immobilized polyethylene oxide (PEO) was achieved using an argon radio-frequency glow discharge treatment (RFGD) of polyethylene films precoated with Brij hydrocarbon-PEO surfactants. Surface wettability of RFGD-treated and washed surfaces increased the most when PEO surfactants with unsaturated and/or long alkyl tails were used. ESCA measurements of treated and washed surfaces showed increases of surface $\mathrm{O} / \mathrm{C}$ ratios and ether carbon peaks in high resolution Cls spectra. These results demonstrate the retention of the PEO surfactants on the treated surfaces. Fibrinogen adsorption on these treated surfaces was significantly reduced, from 500 to $50 \mathrm{ng} / \mathrm{cm}^{2}$, indicating the non-fouling properties of the RFGD-immobilized PEO surfactants.
\end{abstract}

\section{INTRODUCTION}

Protein-repellent or 'non-fouling' surfaces are needed as components of drug delivery vehicles to avoid loss of high-cost peptide and protein drugs. They are also potentially useful as coatings for drug-containing nanoparticles, both to avoid phagocytosis in the liver and also to affect distribution of the particles in different organs. Such surfaces are also important to other biotechnological and medical applications, such as diagnostic assays, biosensors, bioseparations, implants and medical devices. The non-fouling properties of a wide variety of surface treatments have been investigated. ${ }^{1}$ The deposition of compositions containing polyethylene oxide (PEO) has received the most attention. ${ }^{1-8}$ It has been shown that when PEO chains of MW c. 1000 or more are immobilized on a surface, the adsorption of proteins as fibrinogen can be reduced significantly.

In this study, we have developed a novel radiofrequency glow discharge (RFGD) process to bind a PEO-containing surfactant onto a hydrophobic surface. In this process, the surfactant is first 'anchored' on the polymer surface via physical adsorption from a solvent which swells the substrate polymer. Then the solvent is evaporated and the adsorbed surfactant is bound covalently to the surface by a gas discharge treatment, which causes 'crosslinking' of the surfactant with the polymer molecules in the surface. In this paper we will present results obtained with a variety of PEO surfactants, physically adsorbed onto polyethylene surfaces and then bound to the surface with an argon gas discharge.

\section{MATERIALS AND METHODS}

\section{Materials}

Table 1 lists the code names and compositions of the compounds studied in this paper. PEO1K, Brij surfactants (from Sigma Chemical Co.) and Triton X-165 (as a gift from Rohm and Haas Co., Philadelphia, PA) were used as received. Brij99 was selected as a model compound for the adsorption and desorption studies. The low-density polyethylene 
Table 1. PEO surfactants and PEO used in this study

\begin{tabular}{lcccc}
\hline $\begin{array}{l}\text { PEO } \\
\text { surfactant }\end{array}$ & $\begin{array}{c}\text { Chemical } \\
\text { structure }\end{array}$ & $\begin{array}{c}\text { Average } \\
\text { molecular } \\
\text { weight }\end{array}$ & $\begin{array}{c}\text { Average PEO } \\
\text { molecular } \\
\text { weight }\end{array}$ & O/C ratio \\
\hline Brij99 & $\mathrm{C}_{18: 1}-\mathrm{EO}_{20}$ & 1148 & 880 & 0.36 \\
Brij78 & $\mathrm{C}_{18}-\mathrm{EO}_{20}$ & 1150 & 880 & 0.36 \\
Brij35 & $\mathrm{C}_{12}-\mathrm{EO}_{20}$ & 1066 & 880 & 0.40 \\
Triton X-165 & $\mathrm{C}_{8} \mathrm{Ph}_{-}-\mathrm{EO}_{16}$ & 910 & 700 & 0.37 \\
PEO Homopolymer & & & \\
PEO1K & $\left(\mathrm{CH}_{2} \mathrm{CH}_{2} \mathrm{O}\right)_{23}$ & 1000 & 1000 & 0.50 \\
\hline
\end{tabular}

${ }^{a}$ Provided by the manufacturer.

(LDPE) films (Cadillac Plastic, Seattle, WA) were cleaned sequentially with $\mathrm{CHCl}_{3}$, acetone and water for $15 \mathrm{~min}$ each in a sonicator and dried in air. Reagent-grade chemicals were used as received in this study without further purification. Fibrinogen was purified from fresh baboon blood (from the Regional Primate Research Center, Seattle, WA) and radiolabelled with ${ }^{125} \mathrm{I}$ according to previously published protocols. ${ }^{9}$

\section{Adsorption and desorption of Brij99 on LDPE}

Brij99 was chosen as a model surfactant for optimization of the adsorption and washing or desorption steps. A simple dip-coating method was applied to adsorb Brij99 onto LDPE films. LDPE films were dipped in a $1 \% \mathrm{w} / \mathrm{v}$ Brij99 solution, freshly prepared in chloroform, at room temperature for $30 \mathrm{~s}$. Then the coated films were dried in a laminar flow hood for one day at room temperature. Desorption (or washing) of the surfactant from the coated but untreated surfaces was studied in both water and chloroform solvents. After RFGD treatment, treated films were washed with water for 30 min twice and soaked overnight in fresh water before further surface characterization. This process was designed to completely remove all of the physically adsorbed Brij99 from the surface.

\section{Gas discharge treatment}

A capacitive radio-frequency flow discharge at $13.56 \mathrm{MHz}$ (HF-300, ENI Power Systems Inc., Rochester, NY) was used to treat LDPE or LDPE/PEO-surfactant surfaces in a glass cylinder reactor (i.d. $11.5 \mathrm{~cm} \times 80 \mathrm{~cm}) . \Lambda$ static argon $(\Lambda \mathrm{ir}$ Products and Chemicals Inc., Allentown, PA, Pre-pure grade: $>99.95 \%)$ gas discharge without flow was generated at a reactor pressure of
$25 \mu \mathrm{m} \mathrm{Hg}$, a low power of 2.5 or $5 \mathrm{~W}$, and ambient temperature.

\section{Surface analyses}

The RFGD-treated surfaces were characterized by ESCA and water contact-angle measurements. ESCA measurements were done on an SSX-100 spectrometer (Surface Science Instruments, Mountain View, CA) using a monochromatic AlK $\alpha$ Xray source with a $5 \mathrm{eV}$ floodgun. A Ramé-Hart goniometer was used to measure advancing-water contact angles of treated films at room temperature in air.

\section{Fibrinogen adsorption}

Polymcr films $(15 \mathrm{~mm} \times 11 \mathrm{~mm})$ were prehydrated in citrated phosphate buffered saline with $0.02 \%$ sodium azide and $0.01 \mathrm{M}$ sodium iodide (CPBSzi) at $37^{\circ} \mathrm{C}$ for $4 \mathrm{~h}$. Then, an ${ }^{125}$ I-labelled baboon fibrinogen solution was added to reach a $0.2 \mathrm{mg} / \mathrm{ml}$ total protein concentration (average counts $-2 \times 10^{6} \mathrm{cpm} / \mathrm{mg}$ fibrinogen) and the films were incubated at $37^{\circ} \mathrm{C}$ for $2 \mathrm{~h}$. After incubation, the protein adsorbed films were eluted with $100 \mathrm{ml}$ of fresh CPBSzi and counted with a $\gamma$-counter.

\section{RESULTS AND DISCUSSION}

ESCA was used to evaluate the effectiveness of the wash step (Fig. 1). Results indicate that the physically adsorbed Brij99 surfactant is easily removed from the untreated surface by soaking in either chloroform or water for 2 or $5 \mathrm{~min}$, respectively. These results emphasize that simple physical adsorption of the surfactant, even in a swelling solvent such as $\mathrm{CH}_{2} \mathrm{Cl}_{2}$, is not sufficient to bind it to 


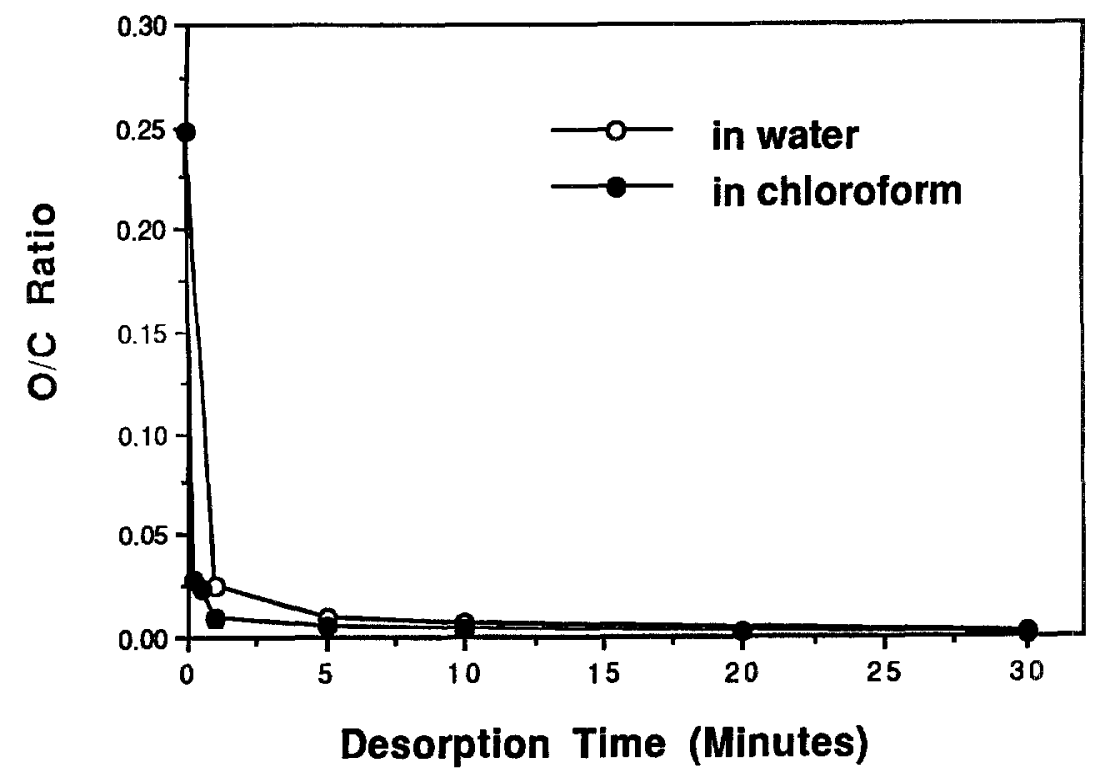

Fig. 1. ESCA O/C ratio shown as a function of the kinetics of desorption (washing) time in water or chloroform for Brij 99 physically adsorbed on untreated LDPE. Brij99 was deposited on LDPE surfaces from a $1 \% \mathrm{w} / \mathrm{v}$ chloroform solution for $30 \mathrm{~s}$ at room temperature, dried for one day and then washed at room temperature in either water of chloroform.

the LDPE surface. ESCA results of RFGD-treated and washed LDPE and LDPE/Brij99 surfaces are shown in Fig. 2. It can be seen that there is an increase of surface $\mathrm{O} / \mathrm{C}$ ratios of $\mathrm{RFGD}$-treated LDPE, indicating that the treatment causes some surface oxidation of LDPE, although the $\mathrm{O} / \mathrm{C}$ ratios still remain significantly less than on the treated surfactant-LDPE films. The surface $O / C$ ratios of $R F G D$-treated LDPE/Brij99 films increase with treatment time up to a plateau at $30 \mathrm{~s}$, indicating the probable presence of PEO surfactant molecules on the treated surfaces when compared to the control LDPE films. Thus, the treatment must bind the surfactant to the substrate surface. The recombination of alkyl and polymer free radicals, formed by the ArRFGD treatment is the most likely explanation of such an immobilization, leading to crosslinking of the PEO surfactant to the polyethylene chains.

Advancing water-contact angles on the treated and washed control LDPE, LDPE/PEO-homopolymer and LDPE/PEO-surfactant films are shown in Fig. 3. The RFGD-treated LDPE surfaces exhibit a slight decrease in water contact

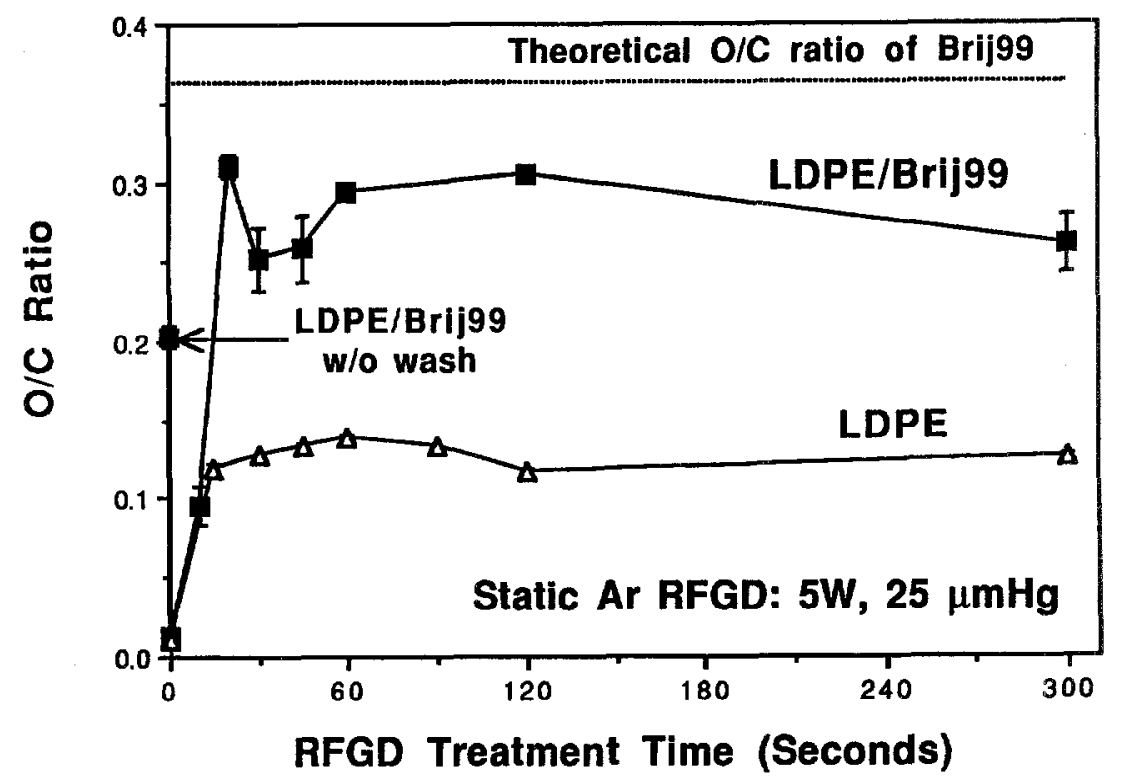

Fig. 2. ESCA O/C ratios for LDPE and LDPE/Brij99 surfaces after Ar RFGD treatment and washing in water. 


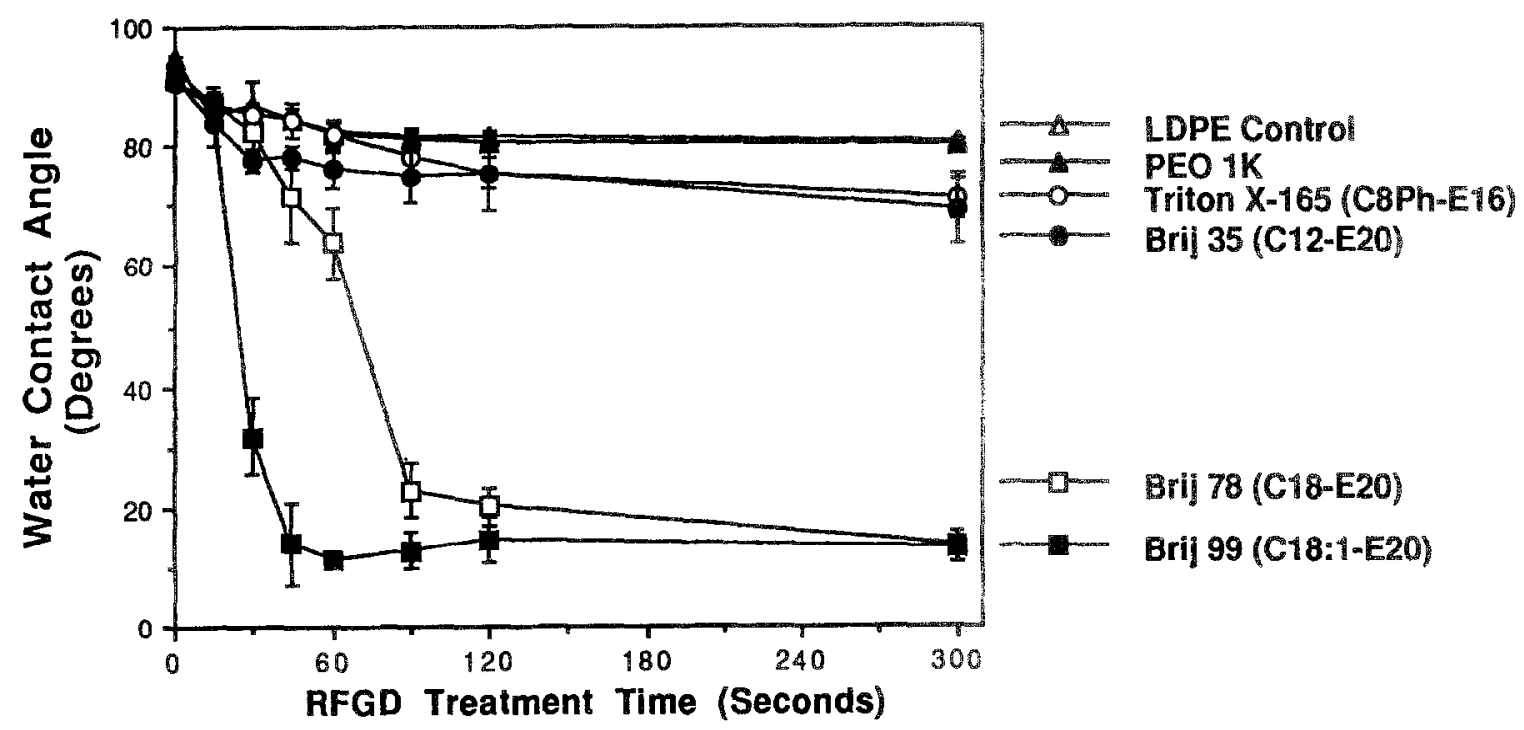

Adsorption: $0.5 \% \mathrm{w} / \mathrm{v}$ in chloroform at R.T. for 30 seconds. Desorption: washed in water for $2 \times 30 \mathrm{~min}$. + overnight.

Fig. 3. Water contact angles on Ar RFGD-treated LDPE and LDPE/PFO-surfactant surfaces after washing and drying.

angles, probably due to surface oxidation, as suggested by the ESCA results. Only surfaces modified with PEO surfactants containing a long unsaturated (Brij99) or saturated alkyl chain (Brij78), show a large reduction of water contact angle $\left(<20^{\circ}\right)$ for treatment times over about $45-90 \mathrm{~s}$. No significant improvement of surface wettability was observed on the surfaces modified with the PEO homopolymer, PEO1K. It can also be seen that other PEO surfactants with a shorter alkyl group (Brij35) or an aryl group (Triton X-165) showed no improvement when compared with the LDPE control. These results support the proposed cross- linking mechanism which should be favored by longer and unsaturated alkyl chains, It has been previously reported that polyethylene forms free radicals which cause surface crosslinking when $P E$ is treated with inert gas plasmas. 10 This process was named CASING (Crosslinking by Active Species of INert Gas). It is likely that the mechanism of the immobilization of PEO surfactants is similar.

Fibrinogen adsorption on RHGD-treated LDPE and LDPE/Brij99 surfaces is shown in Fig. 4. Fibrinogen adsorption on RHGD-treated and washed control LDPE films increases with increase

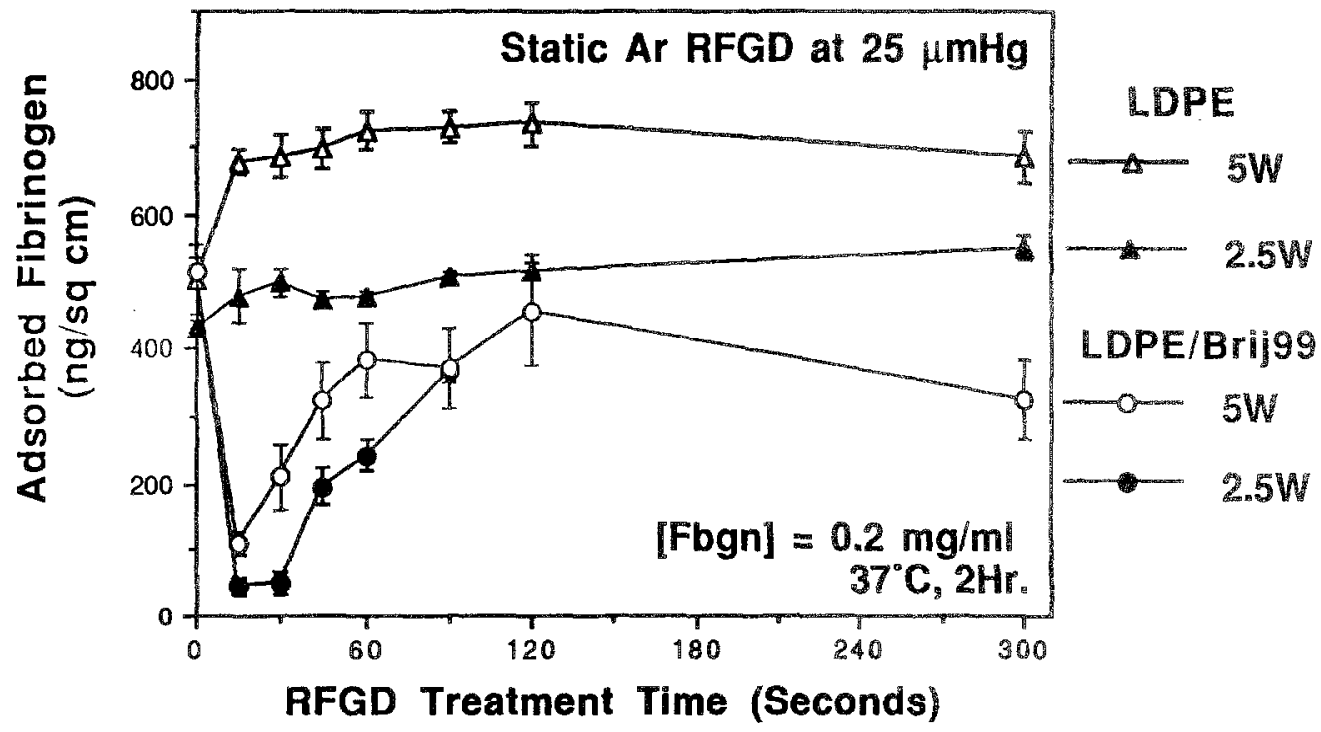

Fig. 4. Fibrinogen adsorption on Ar RFGD-treated LDPE and LDPE/Brij99 surfaces. Results are shown for 2.5 and 5 W plasma power levels. After prehydration of films in CPBSzi buffer for $4 \mathrm{~h}$, protein adsorption was carried out in $0.2 \mathrm{mg} / \mathrm{ml}$ fibrinogen solutions at $37^{\circ} \mathrm{C}$ for $2 \mathrm{~h}$. 
of treatment time as well as treatment power, probably because of the increased surface oxidation. On the other hand, the RFGD-treated LDPE/Brij99 surfaces exhibit a significant reduction of fibrinogen adsorption to about $50 \pm 11$ and $110 \pm 18 \mathrm{ng} / \mathrm{cm}^{2}$ when treated $15 \mathrm{~s}$ with $2 \cdot 5$ and $5 \mathrm{~W}$ Ar plasmas, respectively. These results also support the retention of the Brij99 on the LDPE surface. When RFGD treatment time is prolonged, fibrinogen adsorption increases on the LDPE/Brij99 surfaces, which may be due to the surfacc oxidation and/or degradation of PEO duc to the etching action of the argon plasma. In summary, the best non-fouling surface was obtaincd when LDPE/Brij99 films were treated at a low power level for a short treatment time, c.g. $2.5 \mathrm{~W}$ and $15-30 \mathrm{~s}$. This is a very simple, clean and short process for producing a non-fouling surface.

\section{CONCLUSIONS}

A non-fouling polymer surface, exhibiting very low fibrinogen adsorption, was obtained using a short, low-power Ar RFGD treatment on an LDPE surface which had been precoated with a PEO surfactant. This simple process may have wide applicability for modifying polymer surfaces in general as well as biomaterial surfaces in particular.

\section{ACKNOWLEDGEMENTS}

We thank NIH (GM 40111-02 and 03) and the Washington Technology Center for thcir financial support for this study, and NESAC/BIO at University of Washington (NIH, RR 01296) for the use of the ESCA facility.

\section{REFERENCES}

1. Andrade, J. D., Nagaoka, S., Cooper, S., Okano, T. \& Kim, S. W., Trans ASAIO, 33 (1987) 75.

2. Merrill, E. W. \& Salzman, E. W., ASAIO J., 6 (1983) 60.

3. Mori, Y., Nagaoka, S., Takiuchi, H., Kikuchi, T., Noguchi, N., Tanzawa, H. \& Noishiki, Y., Trans ASAIO, 28 (1982) 459.

4. O'Mullane, J. E., Davison, C. J., Petrak, K. \& Tomlinson, E., Biomaterials, 9 (1988) 338.

5. (a) Gombotz, W. R., Wang, G. H. \& Hoffman, A. S., $J$. Appl. Polym. Sci., 37 (1989) 91

(b) Gombotz, W. R., Wang, G. H. \& Hoffman, A. S., $J$. Biomed. Mater. Res., 25 (1991) 1547.

6. Sun, Y. H., Gombotz, W. R. \& Hoffman, A. S., J. Bioact. \& Compat. Polym., 1 (1986) 316.

7. Okkema, A. Z., Grasel, T. G., Zdrahala, R. J., Solomon, D. D. \& Cooper, S. L., J. Biomater. Sci. Polym. Ed., 1 (1989) 43

8. Lopez, G. P., Ratner, B. D., Tidwell, C. D., Haycox, C. L., Rapoza, R. J. \& Horbett, T. A., J. Biomed. Mater. Res., 26 (1992) 415.

9. Horbett T. A., Cheng, C. M., Ratner, B. D., Hoffman, A. S. \& Hanson, S. R., J. Biomed. Mater. Res. 20 (1986) 739.

10. Hansen, R. H. \& Schonhorn, H., J. Polym. Sci., Polym. Lett. Ed., 4 (1966) 203. 